\title{
28 Research Soure \\ Deep thrombosis characterization using photoacoustic imaging with intravascular light delivery
}

\author{
Yuqi Tang \\ Duke University \\ Huaiyu Wu \\ North Carolina State University \\ Paul Klippel \\ Pennsylvannia State University \\ Bohua Zhang \\ North Carolina State University \\ Hsiao-Ying Shadow Huang \\ North Carolina State University \\ Yun Jing
}

Pennsylvannia State University

Xiaoning Jiang ( $\nabla$ xjiang5@ncsu.edu )

North Carolina State University

Junjie Yao ( $\square$ junjie.yao@duke.edu )

Duke University

\section{Research Article}

Keywords: Thrombosis, photoacoustic tomography, internal light illumination, photoacoustic frequency, retracted blood clot, unretracted blood clot, blood oxygenation

Posted Date: January 31st, 2022

DOI: https://doi.org/10.21203/rs.3.rs-1312641/v1

License: (c) (i) This work is licensed under a Creative Commons Attribution 4.0 International License. Read Full License 


\section{Abstract}

Venous thromboembolism (VTE) is a condition in which blood clots form within the deep veins of the leg or pelvis to cause deep vein thrombosis. The optimal treatment of VTE is determined by thrombus properties such as the age, size, and chemical composition of the blood clots. The thrombus properties can be readily evaluated by using photoacoustic computed tomography (PACT), a hybrid imaging modality that combines the rich contrast of optical imaging and deep penetration of ultrasound imaging. With inherent sensitivity to endogenous chromophores such as hemoglobin, multispectral PACT can provide composition information and oxygenation level in the clots. However, conventional PACT of clots relies on external light illumination, which provides limited penetration depth due to strong optical scattering of intervening tissue. In our study, this depth limitation is overcome by using intravascular light delivery with a thin optical fiber. To demonstrate in vitro blood clot characterization, clots with different acuteness and oxygenation levels were placed underneath ten-centimeter-thick chicken breast tissue and imaged using multiple wavelengths. Acoustic frequency analysis was performed on the received PA channel signals, and oxygenation level was estimated using multispectral linear spectral unmixing. The results show that, with intravascular light delivery, clot oxygenation level can be accurately measured, and the clot age can thus be estimated. In addition, we found that retracted and unretracted clots had different acoustic frequency spectrum. While unretracted clots had stronger high frequency components, retracted clots had much higher low frequency components due to densely packed red blood cells. The PACT characterization of the clots was consistent with the histology results and mechanical tests.

\section{Introduction}

Venous thromboembolism (VTE) is one of the leading causes of death worldwide and accounts for significant morbidity and mortality [1]. Deep vein thrombosis (DVT) may cause life-threatening pulmonary embolism (PE) when clots can break free and the debris enters lung arteries, resulting in sudden death [2]. VTE has affected $300,000-600,000$ patients every year and one-third of DVT cases have turned into PE [3]. The age of the clot affects the level of pulmonary embolism risk and DVT treatment method. Clinically, it has been reported that low recanalization rates and higher number of retrieval attempts are associated with fibrin-rich clots [4]-[8]. It is hypothesized that the mechanical properties of the clots result in different resistance of clots to the thrombolytic treatment: the retracted or fibrin-rich clots are stiffer and denser than the unretracted or erythrocytes-rich clots, and therefore has lower thrombolysis efficiency [6], [9].

Thrombolysis treatment is usually associated with anticoagulation therapy to avoid the risk of recurrence and bleeding during continuous therapy. Thus, a diagnostic method is needed for determining the properties of the clots [10]-[13]. In addition, the optimal treatment of VTE is determined by thrombus factors such as the age, size, and chemical composition of the blood clots. Currently, ultrasound (US) imaging is widely used due to its non-invasiveness, low cost and high spatial-temporal resolution. To assess the venous thrombosis, the most common tests are B-mode or Doppler US imaging. However, Bmode US imaging may suffer from low sensitivity for deep vein detection while Doppler US imaging relies 
on the blood flow [14]-[16]. Besides, neither B-mode nor Doppler US imaging can quantitatively measure the mechanical information of clots, and thus cannot characterize the clot stage and provide effective guidance for treatment [17]. Quantitative US shear wave elasticity (SWE) imaging has recently been proposed as an alternative method for clot characterization [17]-[21]. Yet, most of the SWE methods have not considered the tissue inhomogeneity, in which the boundary conditions may affect the shear wave transmission and reflection and lead to inaccurate measurement [18], [22].

Photoacoustic computed tomography (PACT), a hybrid imaging modality that combines the rich contrast of optical absorption and deep penetration of ultrasound detection, have been widely used for disease diagnosis and surgical guidance [23]-[31]. PACT's capability of deep-tissue functional imaging can be readily used for thrombus factor estimation. When the photons from a pulsed laser are diffused in tissue and absorbed by either endogenous or exogenous chromophores, the absorbed optical energy is completely or partially converted to heat, resulting in transient temperature rise that leads to thermoelastic expansion and pressure rise. The pressure propagates in the tissue as ultrasound waves, which can be detected outside the tissue by an ultrasound transducer array to form a tomographic image of the optical absorption. The spatial resolution of the system depends on the central frequency, bandwidth, and aperture size of the transducer array, and the imaging frame rate is determined by the pulse repetition rate of the laser [32], [33]. In PACT, a target with a smaller dimension or a higher stiffness (i.e., higher speed of sound) has a shorter PA signal duration, corresponding to higher frequency components [34]. Due to PACT's inherent sensitivity toward endogenous chromophores such as hemoglobin, multispectral PACT can provide composition information and oxygenation level in blood clots, which can potentially be used for characterizing clot's type and age. As the acoustic frequency spectrum of the PA signal is related to the object size or stiffness, the structural or mechanical information of clots can be extracted from the frequency spectrum of the PA signal [34]. Das et al. have demonstrated the feasibility of PA clot imaging as well as sonothrombolysis treatment monitoring in clear medium [35], [36]. However, there has been no clot characterization using PA imaging in deep tissue, limited by the external light illumination in conventional PACT. There is only a few reported works on USguided intravenous sonothrombolysis [8], [9], [37]. As deep veins are typically several centimeters away from the skin surface, external light illumination provides limited imaging depth due to strong optical scattering of tissue.

In this work, we have overcome the imaging depth problem of the conventional PACT by using internal light delivery, as demonstrated previously [38]. Blood clots with different oxygenation levels were imaged underneath ten-centimeter-thick chicken breast tissue. PA channel data was used for structural analysis of the clots, and the reconstructed PA images were used for clot oxygenation level estimation. The PACT results were then compared with the measurements from uniaxial tensile tests and Hematoxylin and Eosin (H\&E) histology analysis. The results have showed a strong correlation between the PA characterization of the blood clots and their mechanical stiffness, demonstrating the potential of our approach to provide imaging guidance of thrombosis treatment. 


\section{Methods}

PACT system: The PACT system is based on a commercial ultrasound scanner (Vantage 256, Verasonics, Kirkland, Washington) with a linear array transducer (L7-4, Philips ATL, Atlanta, Georgia) (Fig. 1a). The linear array transducer has 128 elements, a central frequency of $5 \mathrm{MHz}$, and a $60 \%$ bandwidth. Laser pulses at $700 \mathrm{~nm}$ and $800 \mathrm{~nm}$ with a pulse energy of $13 \mathrm{~mJ}$ and $11 \mathrm{~mJ}$ were delivered through a multimode fiber (FT1500UMT, Thorlabs, Newton, New Jersey) with a numerical aperture of 0.39 and core diameter of $1.5 \mathrm{~mm}$. The distal end of the fiber was fused with a lab-made catheter, which consisted of an air-filled cavity and a plano-concave lens (Edmund, Barrington, New Jersey) (Fig. 1b). The lens had an aperture size of $3 \mathrm{~mm}$ and a back focal length of $-6.56 \mathrm{~mm}$. The optical beam profile with and without the catheter was simulated using Zemax, and the cavity dimension was optimized based on the simulation results. The catheter has two functions: (1) With water as the surrounding medium, it provides a larger refractive index mismatch at the distal end of the fiber, and thus delivers beams with high divergence to cover a large area of the clot; (2) It separates the blood or clot debris from the fiber surface to avoid any contamination and generation of "hot spots" that can potentially damage the fiber.

Blood clot preparation: The blood clots were prepared based on a similar protocol from our previous work [39]. Bovine blood (Lampire, Pipesville, Pennsylvania) was mixed with $2.75 \% \mathrm{~W} / \mathrm{V} \mathrm{CaCl}_{2}$ solution (Fisher Scientific, Hampton, New Hampshire) with a volume ratio of 10:1 for coagulation. To change the oxygen saturation of hemoglobin $\left(\mathrm{sO}_{2}\right)$, additional $\mathrm{NaHCO}_{3}(37 \mathrm{mg} / \mathrm{ml}$ of blood $)$ or $\mathrm{Na}_{2} \mathrm{~S}_{2} \mathrm{O}_{4}(2.5 \mathrm{mg} / \mathrm{ml}$ of blood) was added to the blood- $\mathrm{CaCl}_{2}$ solution, followed by 5-min oxygen or carbon dioxide perfusion [40], [41]. After mixing, the blood was transferred to either borosilicate or flint Pasteur pipets (Fisher Scientific, Hampton, New Hampshire) and immersed in a $37^{\circ} \mathrm{C}$ water bath for 3 hours. After clot formation, the tubes were stored at $4^{\circ} \mathrm{C}$ for 72 hours to induce clot retraction. Figure 1c shows a comparison between borosilicate (retracted) and flint (unretracted) clot [42].

PA imaging and data analysis: For PA imaging, the clot was extracted from the Pasteur pipets, rinsed with PBS, and placed around $5 \mathrm{~mm}$ away from the catheter inside a PBS-filled Tygon tube (4.76 mm ID). To demonstrate the deep tissue clot characterization with our PACT system, around ten-centimeter-thick chicken tissue was placed between the transducer and the Tygon tube (Fig. 1a). For each clot, 300 frames of two-dimensional PA data were acquired at each optical wavelength, and the images were reconstructed with both delay-and-sum (DAS) and delay-multiply-and-sum (DMAS) algorithms [43], [44]. While DAS preserves the linear dependence of the PA signals on the optical absorption, it often suffers from the strong limited-view reconstruction artifacts. DMAS is able to suppress the reconstruction artifacts, but it is a nonlinear process and cannot be used for $\mathrm{sO}_{2}$ estimation. Therefore, we combined the DAS and DMAS in our data processing. We used the DMAS image as a binary mask to reduce the artifacts in the DAS image. The masked DAS images after 300-frame-averaging were then used for $\mathrm{sO}_{2}$ estimation using the linear spectral unmixing method based on the absorption spectra of oxy-and deoxyhemoglobin (Fig. 1d) [45]-[47]. We also investigated the acoustic frequency spectrum of the clot. To 
minimize the impact of the catheter signals, channel data from the transducer elements directly above the clot were extracted and processed with Fourier transform.

\section{Histology examination of the clots}

As the frequency components of the PA signal depend on the object size and stiffness, we compared our frequency spectrum with the histology results. Hematoxylin and Eosin (H\&E) staining was conducted on all the clot samples imaged by PACT. After clot formation and retraction, the samples were fixed in $10 \%$ neutralized buffered formalin. The formalin fixed clot samples were cut into 5 - $\mu$ m-thick slices and stained with H\&E. The slides were scanned using an EVOS compound light microscope (EVOS®FL Auto Imaging System, Life Technologies Corporation, Carlsbad, CA, USA) and images were stored digitally. The ImageJ software (National Institutes of Health, Bethesda, MD, USA) and a custom processing algorithm (MATLAB R2018b, MathWorks, MA, USA) were used for the quantitative analysis with color-based segmentation. The retraction of the clot was estimated from the area percentage of the erythrocytes region in the clot histology images and was related to PA frequency spectrum analysis.

\section{Measurement of the clot stiffness}

Stiffness of the blood clots was tested with BioTester 5000 (CellScale, Waterloo, Ontario, Canada). Two rakes with five tungsten tines each were used at the two ends of the cylindrical clot in the longitudinal directions. Each group of the five tines were equally distributed along a sample dimension of $4.5 \mathrm{~mm}$ during the test. For each group of clots, three samples were tested with an average initial length of $6 \mathrm{~mm}$. The samples were then subject to loading under $10 \%, 25 \%$ and $40 \%$ strain conditions for three loops, corresponding to a displacement of $600 \mu \mathrm{m}, 1500 \mu \mathrm{m}$ and $2400 \mu \mathrm{m}$, respectively. The force-displacement curve was recorded. Curve fitting was applied based on the linear phase of the force-displacement and the Young's modulus was estimated as follows,

$$
E=\frac{\sigma}{\epsilon}=\frac{F / A}{\Delta l / l}
$$

in which $\varepsilon$ is the strain in the sample and $\sigma$ is the stress. $\sigma$ was acquired from the applied force $F$ and the cross-section area $A$ of the sample. / is the initial length of the clot and $\Delta /$ is the displacement under the applied force.

\section{Results}

\section{PACT of the blood clots in deep tissue}

The fraction power on the plano-concave lens at different air cavity lengths $d$ is shown in Fig. 2a. Limited by the radius of the lens $(1.5 \mathrm{~mm})$, air cavity with length longer than $7 \mathrm{~mm}$ results in a power loss of more than $10 \%$. The simulated internal illumination beam profiles with and without the plano-concave lens are shown in Fig. $\mathbf{2 b}$. We extracted the beam profile $5 \mathrm{~mm}$ from the distal end of the catheter, i.e., $\mathrm{L}=5 \mathrm{~mm}$. The length of the air cavity $d$ was varied from $1 \mathrm{~mm}$ to $7 \mathrm{~mm}$ in the simulation to achieve an optimized beam size. Without the concave lens, the beam slowly diverged (FWHM radius $=0.5 \mathrm{~mm}$ with $\mathrm{d}=7 \mathrm{~mm}$ ). 
With the concave lens, the beam diverged faster and can cover a larger region of the clot (FWHM radius = $1 \mathrm{~mm}$ with $\mathrm{d}=7 \mathrm{~mm}$ ). The clots were imaged with multispectral PA imaging at 700 and $800 \mathrm{~nm}$. Internal illumination was provided by placing the catheter around $5 \mathrm{~mm}$ from the clot surface. Images reconstructed with DAS have strong streak artifacts due to the limited detection angle (Fig. 2c). Images reconstructed with DMAS have reduced artifacts and improved contrast (Fig. 2d). As clots had high optical scattering and absorption coefficients with densely packed red blood cells (RBCs), a penetration depth of $5 \mathrm{~mm}$ into the clot can be achieved from the reconstructed images. The B-mode US image (Fig. 2e) shows the Tygon tube was placed underneath ten-centimeter-thick chicken breast tissue. The merged US and PA image is shown in Fig. 2f, highlighting the deep imaging depth achieved by the internal light illumination.

\section{Estimating the clot oxygenation level}

The $\mathrm{sO}_{2}$ of the clot should be affected by the initial oxygenation level of blood. $\mathrm{sO}_{2}$ was estimated with DMAS-masked PA images acquired at 700 and $800 \mathrm{~nm}$. Optical fluence was compensated for based on the measured pulse energy during the experiment. We did not observe any difference in $\mathrm{sO}_{2}$ between flint and borosilicate clots made from the native blood without either $\mathrm{NaHCO}_{3}$ or $\mathrm{Na}_{2} \mathrm{~S}_{2} \mathrm{O}_{4}$ (Figs. 3a and 3d). $\mathrm{NaHCO}_{3}$ increased the blood $\mathrm{pH}$ and shifted the oxyhemoglobin dissociation curve to the left, therefore increased $\mathrm{sO}_{2}$ with $\mathrm{O}_{2}$ infusion (Figs. $3 \mathrm{~b}$ and $3 \mathrm{e}$ ). $\mathrm{Na}_{2} \mathrm{~S}_{2} \mathrm{O}_{4}$ chemically reduced the oxygenated hemoglobin to deoxygenated hemoglobin by reacting with oxygen and water to form soluble acidic products. Therefore, clots formed from blood with $\mathrm{Na}_{2} \mathrm{~S}_{2} \mathrm{O}_{4}$ had much lower $\mathrm{sO}_{2}$ levels (Figs. $3 \mathrm{c}$ and $3 \mathrm{f}$ ).

Analyzing the acoustic frequency spectrum of the clots: While the reconstructed $\mathrm{PA}$ images and $\mathrm{sO}_{2}$ estimation can provide the general morphological and functional information of the clots, the internal structure of the clots is related to the acoustic frequency spectrum of PA channel data. Smaller or stiffer targets generate PA signals with higher frequency components, and vice versa. The previous studies have shown that the container material affects the retraction of the blood clot [34]. We found that clots prepared with flint and borosilicate glass had relatively homogenous internal structure (Figs. 4a and 4d). While borosilicate glass led to retracted clots, flint glass favored the formation of less retracted or unretracted clots. The clot retraction was reflected by the packing density of RBCs in histology. The densely packed RBCs formed relatively large internal structures (Figs. 4b-c) and manifested as low frequency components in the PA frequency spectrum (Fig. 4g). The loosely packed RBCs formed relatively small internal structures and produced high frequency components in the PA frequency spectrum. Moreover, we estimated the occupation percentage of the clot signals in the histology images. We found that an occupation percentage of $>90 \%$ corresponded to low frequency components of $<2 \mathrm{MHz}$, $80-90 \%$ to medium frequency components of $2-4 \mathrm{MHz}$, and $<80 \%$ to high frequency components of $>4$ $\mathrm{MHz}$ (Fig. 4h).

We also investigated the impact of $\mathrm{NaHCO}_{3}$ or $\mathrm{Na}_{2} \mathrm{~S}_{2} \mathrm{O}_{4}$ on the internal structures of the clots. As the blood $\mathrm{pH}$ affects the fibrin conversion from fibrinogens, low $\mathrm{pH}$ environment favors the formation of retracted clot and high $\mathrm{pH}$ environment favors less retracted clot [48]. In addition, borosilicate glass has 
negative surface charges and can initiate fast fibrin formation adjacent to the surface [48], [49]. $\mathrm{NaHCO}_{3}$ has been shown to improve the hydrophilicity of the surface and subsequently improve the adsorption of protein [50]. Both pH and surface material's effect on clot retractiveness have been studied separately, but no study has done on the combined effect. Therefore, it is challenging to accurately attribute the structures of the clots to specific factors. Nevertheless, we observed inhomogeneous structures formed inside the clots when either $\mathrm{NaHCO}_{3}$ or $\mathrm{Na}_{2} \mathrm{~S}_{2} \mathrm{O}_{4}$ was mixed with blood (Figs. 5 and 6). All three types of RBC packing density were observed in clots from both borosilicate and flint Pasteur pipets: high (Figs. 5a, $5 d, 6 a$, and $6 d$ ), medium (Figs. 5b, 5e, 6b, and 6e), and low (Figs. 5c, 5f, and 6c).

For clots from blood with $\mathrm{NaHCO}_{3}$, the PA frequency spectrum had a wide range with strong low, medium, and high frequency components, for both types of glass used (Fig. 5g). After examining the histology, we found that the RBCs were more densely packed near the clot edges, which were in close contact with the Pasteur pipets. The regions with low RBC density were mainly located in deeper regions of the clots. Therefore, we believe the heterogeneous structure of $\mathrm{NaHCO}_{3}$ clot may be attributed to the increased hydrophilicity of the pipet surface and increased $\mathrm{pH}$. For clots from blood with $\mathrm{Na}_{2} \mathrm{~S}_{2} \mathrm{O}_{4}$, the PA frequency spectrum was also wider than that without $\mathrm{NaHCO}_{3}$ or $\mathrm{Na}_{2} \mathrm{~S}_{2} \mathrm{O}_{4}$, but narrower than that with $\mathrm{NaHCO}_{3}$ (Fig. 6f), which was possibly because the amount of chemical was lower, and the surface hydrophilicity was not significantly changed. While the mechanism of the complex clot structures is out of the scope of this work, the structural information was consistent between the histology results and the PA frequency analysis. The occupation percentage of the RBCs is summarized in Figs. $5 \mathbf{h}$ and $\mathbf{6 g}$.

Measuring the Young's modulus of the clots: Figure 7b shows the measured Young's modulus for each group of the clot samples. The Young's modulus for the clot ranged from 5 to $15 \mathrm{kPa}$, which is comparable with previously reported works [51]. For the clots formed in the borosilicate glass, the Young's modulus showed a $129 \%\left(\mathrm{NaHCO}_{3}\right), 76 \%$ (no chemical) and $51 \%\left(\mathrm{Na}_{2} \mathrm{~S}_{2} \mathrm{O}_{4}\right)$ increase compared with that in the flint glass. Therefore, the clots from the borosilicate glass were clearly more retracted and stiffer compared with those in the flint glass. With the flint glass, the clots from blood with $\mathrm{NaHCO}_{3}$ showed a $36 \%$ drop in Young's modulus compared with normal samples, while the clots from blood with $\mathrm{Na}_{2} \mathrm{~S}_{2} \mathrm{O}_{4}$ showed a $23 \%$ increase. Similarly, with the borosilicate glass, the $\mathrm{NaHCO}_{3}$ samples presented a $17 \%$ decrease and the $\mathrm{Na}_{2} \mathrm{~S}_{2} \mathrm{O}_{4}$ samples presented a $6 \%$ increase in Young's modulus compared with normal samples. The results indicate that our deoxygenation process with $\mathrm{Na}_{2} \mathrm{~S}_{2} \mathrm{O}_{4}$ may increase the Young's modulus during the in vitro clot preparation, while our oxygenation process with $\mathrm{NaHCO}_{3}$ may decrease the Young's modulus.

\section{Discussion}

In this paper, we have demonstrated that PA imaging is capable of structural and functional characterization of blood clots in deep tissue. We used PACT for clots imaging, $\mathrm{sO}_{2}$ estimation, and frequency-based analysis. The results were compared with histology analysis and the Young's modulus measurements. The $\mathrm{SO}_{2}$ and the retraction of the clots could be modified by adding chemicals and 
altering the material for clotting surfaces. We found that retracted clots with RBC-rich structure were associated with low-frequency PA signals and was likely due to the relatively homogeneous bulk structures formed by densely packed RBCs. Similarly, unretracted clots with low RBC density had stronger high-frequency components due to the presence of relatively small RBC clutters. In addition, we found that retraction of the clots was related to fibrin concentration. Therefore, we conclude that the lowfrequency PA signals were associated with the Young's modulus and thus stiffness of the clot.

Similar to US imaging, PA imaging in deep tissues suffers from frequency dependent acoustic attenuation. High frequency signals get more attenuation compared to low frequency signals, leading to less accurate structural characterization based on frequency analysis. For this study specifically, we consider the frequency components below $2 \mathrm{MHz}$ correspond to the presence of retracted structures, and frequency components above $4 \mathrm{MHz}$ correspond to unretracted structures. As the imaging depth changes, the correlation between the frequency components and the clot structures needs to be updated. Nevertheless, we expect this correlation follows a similar pattern that low frequency components indicate retracted structures, and high frequency components indicate unretracted structures.

We also assumed homogeneous fluence distribution within the clots, which is not accurate as clots are highly absorbing and scattering. The assumption, however, does not significantly affect the $\mathrm{sO}_{2}$ estimation, as the 700 and $800 \mathrm{~nm}$ light share similar fluence distribution within the clot. By contrast, the heterogeneous fluence distribution should have a great impact on the frequency-based structure analysis, as the fluence decreases quickly with propagation depth and the frequency spectrum is dominated by the PA signals from the clot surface [52].

The blood clots in this work were prepared with the whole blood, and no centrifuge was applied to separate different blood components [7], [53]. Therefore, the effect of the RBC concentration on clot structure and stiffness was not discussed quantitatively. Limited by the resolution of the optical microscopy, it is challenging to distinguish fibrin matrix and RBCs [53]. As a result, in the H\&E histology analysis, only the RBCs regions were measured without considering the fibrin content, and thus we could not identify the clot samples as erythrocytes-rich or fibrin-rich. Yet, our results showed high consistency in the stiffness measurement, the H\&E histology analysis, and the PA imaging results, demonstrating the potential of using PA imaging for clot characterization.

Our current PA characterization of clots was based on the hemoglobin signals. Additional optical wavelengths can be incorporated for imaging fibrin and other chromophores. To further establish the relationship between the PA imaging and the properties of clots, clots with different ages and RBC concentrations can be used. To demonstrate the clinical applications, clot samples from DVT patients will be used for future studies. Our previous work on a carbon black laser-generated focused ultrasound (LGFU) transducer has shown effective in-vitro sonothrombolysis involving microbubble contrast agent. Our intravascular catheter can be readily combined with the LGFU transducer to provide more efficient treatment and achieve real-time monitoring by providing the clot composition, oxygenation, and structural 
information [54]. All in all, we expect that PA imaging can provide accurate clot characterization in deep tissues, and thus improve the thrombolysis treatment [39].

\section{Declarations}

Funding: This work was sponsored by American Heart Association Collaborative Sciences Award (18CSA34080277); The United States National Institutes of Health (NIH) grants R21EB027981, RF1 NS115581 (BRAIN Initiative), R01 NS111039, R01 EB028143; and Chan Zuckerberg Initiative Grant (2020226178), all to J. Yao; R21EB027304 and R01HL141967, all to X. Jiang.

Competing interest: The authors have no relevant financial or non-financial interest to disclose.

Author contributions: All authors contributed to the study conception and design. Material preparation, data collection and analysis were performed by Yuqi Tang, Huaiyu Wu, Paul Klippel and Bohua Zhang. The first draft of the manuscript was written by Yuqi Tang and Huaiyu Wu and all authors commented on previous version of the manuscript. All authors read and approved the final manuscript.

\section{References}

1. B. Furie and B. C. Furie, "Mechanisms of Thrombus Formation," New England Journal of Medicine, vol. 359, no. 9, pp. 938-949, Aug. 2008, doi: 10.1056/NEJMra0801082.

2. M. G. Beckman, W. C. Hooper, S. E. Critchley, and T. L. Ortel, “Venous Thromboembolism: A Public Health Concern," American Journal of Preventive Medicine, vol. 38, no. 4, Supplement, pp. S495S501, Apr. 2010, doi: 10.1016/j.amepre.2009.12.017.

3. C. M. Bulger, C. Jacobs, and N. H. Patel, "Epidemiology of acute deep vein thrombosis," Techniques in Vascular and Interventional Radiology, vol. 7, no. 2, pp. 50-54, Jun. 2004, doi: 10.1053/j.tvir.2004.02.001.

4. M. Berndt et al., "Thrombus Histology of Basilar Artery Occlusions: Are There Differences to the Anterior Circulation?," Clin Neuroradiol, vol. 31, no. 3, pp. 753-761, Sep. 2021, doi: 10.1007/s00062020-00964-5.

5. P. Moftakhar et al., "Density of thrombus on admission CT predicts revascularization efficacy in large vessel occlusion acute ischemic stroke," Stroke, vol. 44, no. 1, pp. 243-245, Jan. 2013, doi: 10.1161/STROKEAHA.112.674127.

6. K. Maekawa et al., "Erythrocyte-Rich Thrombus Is Associated with Reduced Number of Maneuvers and Procedure Time in Patients with Acute Ischemic Stroke Undergoing Mechanical Thrombectomy," Cerebrovasc Dis Extra, vol. 8, no. 1, pp. 39-49, 2018, doi: 10.1159/000486042.

7. G. M. Gunning, K. McArdle, M. Mirza, S. Duffy, M. Gilvarry, and P. A. Brouwer, "Clot friction variation with fibrin content; implications for resistance to thrombectomy," J Neurointerv Surg, vol. 10, no. 1, pp. 34-38, Jan. 2018, doi: 10.1136/neurintsurg-2016-012721. 
8. L. Goel and X. Jiang, "Advances in Sonothrombolysis Techniques Using Piezoelectric Transducers," Sensors, vol. 20, no. 5, Art. no. 5, Jan. 2020, doi: 10.3390/s20051288.

9. L. Goel et al., "Nanodroplet-mediated catheter-directed sonothrombolysis of retracted blood clots," Microsyst Nanoeng, vol. 7, no. 1, pp. 1-7, Jan. 2021, doi: 10.1038/s41378-020-00228-9.

10. P. M. Ridker et al., "Long-Term, Low-Intensity Warfarin Therapy for the Prevention of Recurrent Venous Thromboembolism," New England Journal of Medicine, vol. 348, no. 15, pp. 1425-1434, Apr. 2003, doi: 10.1056/NEJMoa035029.

11. P. Prandoni et al., "The risk of recurrent venous thromboembolism after discontinuing anticoagulation in patients with acute proximal deep vein thrombosis or pulmonary embolism. A prospective cohort study in 1,626 patients," Haematologica, vol. 92, no. 2, pp. 199-205, Feb. 2007, doi: 10.3324/haematol.10516.

12. M. A. Rodger et al., "Identifying unprovoked thromboembolism patients at low risk for recurrence who can discontinue anticoagulant therapy," CMAJ, vol. 179, no. 5, pp. 417-426, Aug. 2008, doi: 10.1503/cmaj.080493.

13. A. G et al., "Extended oral anticoagulant therapy after a first episode of pulmonary embolism," Annals of internal medicine, vol. 139, no. 1, Jul. 2003, doi: 10.7326/0003-4819-139-1-200307010-00008.

14. H. Heijboer, L. M. Jongbloets, H. R. Büller, A. W. Lensing, and J. W. ten Cate, "Clinical utility of realtime compression ultrasonography for diagnostic management of patients with recurrent venous thrombosis," Acta Radiol, vol. 33, no. 4, pp. 297-300, Jul. 1992.

15. H.-J. Baarslag, E. J. R. van Beek, M. M. W. Koopman, and J. A. Reekers, "Prospective study of color duplex ultrasonography compared with contrast venography in patients suspected of having deep venous thrombosis of the upper extremities," Ann Intern Med, vol. 136, no. 12, pp. 865-872, Jun. 2002, doi: 10.7326/0003-4819-136-12-200206180-00007.

16. D. Gaitini, "Current approaches and controversial issues in the diagnosis of deep vein thrombosis via duplex Doppler ultrasound," Journal of Clinical Ultrasound, vol. 34, no. 6, pp. 289-297, 2006, doi: 10.1002/jcu.20236.

17. E. Mfoumou, J. Tripette, M. Blostein, and G. Cloutier, "Time-dependent hardening of blood clots quantitatively measured in vivo with shear-wave ultrasound imaging in a rabbit model of venous thrombosis," Thromb Res, vol. 133, no. 2, pp. 265-271, Feb. 2014, doi: 10.1016/j.thromres.2013.11.001.

18. X. Liu, N. Li, and C. Wen, "Effect of pathological heterogeneity on shear wave elasticity imaging in the staging of deep venous thrombosis," PLOS ONE, vol. 12, no. 6, p. e0179103, Jun. 2017, doi: 10.1371/journal.pone.0179103.

19. K. J. Haworth, C. R. Weidner, T. A. Abruzzo, J. T. Shearn, and C. K. Holland, “Mechanical properties and fibrin characteristics of endovascular coil-clot complexes: relevance to endovascular cerebral aneurysm repair paradigms," J Neurointerv Surg, vol. 7, no. 4, pp. 291-296, Apr. 2015, doi: 10.1136/neurintsurg-2013-011076. 
20. C.-C. Huang, P.-Y. Chen, and C.-C. Shih, "Estimating the viscoelastic modulus of a thrombus using an ultrasonic shear-wave approach," Med Phys, vol. 40, no. 4, p. 042901, Apr. 2013, doi: 10.1118/1.4794493.

21. S. R. Aglyamov et al., "Young's modulus reconstruction for elasticity imaging of deep venous thrombosis: animal studies," in Medical Imaging 2004: Ultrasonic Imaging and Signal Processing, Apr. 2004, vol. 5373, pp. 193-201. doi: 10.1117/12.539454.

22. M. L. Palmeri and K. R. Nightingale, "What challenges must be overcome before ultrasound elasticity imaging is ready for the clinic?," Imaging Med, vol. 3, no. 4, pp. 433-444, Aug. 2011, doi: 10.2217/iim.11.41.

23. Y. Yan et al., "Photoacoustic-guided endovenous laser ablation: Characterization and in vivo canine study," Photoacoustics, vol. 24, p. 100298, 2021, doi: 10.1016/j.pacs.2021.100298.

24. W. S. Tummers et al., "Intraoperative Pancreatic Cancer Detection using Tumor-Specific Multimodality Molecular Imaging," Ann Surg Oncol, vol. 25, no. 7, pp. 1880-1888, 2018, doi: 10.1245/s10434-018-6453-2.

25. S. A. Ermilov et al., "Laser optoacoustic imaging system for detection of breast cancer," JBO, vol. 14, no. 2, p. 024007, Mar. 2009, doi: 10.1117/1.3086616.

26. M. Toi et al., "Visualization of tumor-related blood vessels in human breast by photoacoustic imaging system with a hemispherical detector array," Sci Rep, vol. 7, no. 1, p. 41970, 2017, doi: 10.1038/srep41970.

27. E. I. Neuschler et al., "A Pivotal Study of Optoacoustic Imaging to Diagnose Benign and Malignant Breast Masses: A New Evaluation Tool for Radiologists," Radiology, vol. 287, no. 2, pp. 398-412, 2018, doi: 10.1148/radiol.2017172228.

28. S.-R. Kothapalli, T.-J. Ma, S. Vaithilingam, Ö. Oralkan, B. T. Khuri-Yakub, and S. S. Gambhir, “Deep Tissue Photoacoustic Imaging Using a Miniaturized 2-D Capacitive Micromachined Ultrasonic Transducer Array," IEEE Transactions on Biomedical Engineering, vol. 59, no. 5, pp. 1199-1204, 2012, doi: 10.1109/TBME.2012.2183593.

29. I. Steinberg, D. M. Huland, O. Vermesh, H. E. Frostig, W. S. Tummers, and S. S. Gambhir, "Photoacoustic clinical imaging," Photoacoustics, vol. 14, pp. 77-98, 2019, doi: 10.1016/j.pacs.2019.05.001.

30. B. Park et al., "A photoacoustic finder fully integrated with a solid-state dye laser and transparent ultrasound transducer," Photoacoustics, vol. 23, p. 100290, 2021, doi: 10.1016/j.pacs.2021.100290.

31. C. Lee, W. Choi, J. Kim, and C. Kim, "Three-dimensional clinical handheld photoacoustic/ultrasound scanner," Photoacoustics, vol. 18, p. 100173, 2020, doi: 10.1016/j.pacs.2020.100173.

32. M. Xu and L. V. Wang, "Universal back-projection algorithm for photoacoustic computed tomography," Phys. Rev. E, vol. 71, no. 1, p. 016706, Jan. 2005, doi: 10.1103/PhysRevE.71.016706.

33. L. V. Wang, "Tutorial on Photoacoustic Microscopy and Computed Tomography," IEEE Journal of Selected Topics in Quantum Electronics, vol. 14, no. 1, pp. 171-179, Jan. 2008, doi: 10.1109/JSTQE.2007.913398. 
34. J. Xia, J. Yao, and L. V. Wang, “Photoacoustic tomography: principles and advances," Electromagn Waves (Camb), vol. 147, pp. 1-22, 2014.

35. D. Das and M. Pramanik, "Combined ultrasound and photoacoustic imaging of blood clot during microbubble-assisted sonothrombolysis," J Biomed Opt, vol. 24, no. 12, p. 121902, Dec. 2019, doi: 10.1117/1.JBO.24.12.121902.

36. D. Das, K. Sivasubramanian, P. Rajendran, and M. Pramanik, "Label-free high frame rate imaging of circulating blood clots using a dual modal ultrasound and photoacoustic system," J Biophotonics, vol. 14, no. 3, p. e202000371, Mar. 2021, doi: 10.1002/jbio.202000371.

37. S. Kutty et al., "Sonothrombolysis of Intra-Catheter Aged Venous Thrombi Using Microbubble Enhancement and Guided Three-Dimensional Ultrasound Pulses," Journal of the American Society of Echocardiography, vol. 23, no. 9, pp. 1001-1006, Sep. 2010, doi: 10.1016/j.echo.2010.06.024.

38. M. Li, B. Lan, W. Liu, J. Xia, and J. Yao, "Internal-illumination photoacoustic computed tomography," JBO, vol. 23, no. 3, p. 030506, Mar. 2018, doi: 10.1117/1.JBO.23.3.030506.

39. J. Kim et al., "Intravascular forward-looking ultrasound transducers for microbubble-mediated sonothrombolysis," Scientific Reports, vol. 7, no. 1, p. 3454, Jun. 2017, doi: 10.1038/s41598-01703492-4.

40. A. Bjørnerud, K. Briley-Sæbø, L. O. Johansson, and K. E. Kellar, "Effect of NC100150 injection on the $1 \mathrm{H}$ NMR linewidth of human whole blood ex vivo: Dependency on blood oxygen tension," Magnetic Resonance in Medicine, vol. 44, no. 5, pp. 803-807, Nov. 2000, doi: 10.1002/15222594(200011)44:5<803::AID-MRM19>3.0.C0;2-K.

41. B. Seaton and B. B. Lloyd, "The effects of $\mathrm{pH}$ on the equilibrium constants of various models for the haemoglobin-oxygen equilibrium in vitro," Respiration Physiology, vol. 20, no. 2, pp. 209-230, Mar. 1974, doi: 10.1016/0034-5687(74)90108-X.

42. J. T. Sutton, N. M. Ivancevich, S. R. Perrin, D. C. Vela, and C. K. Holland, "Clot retraction affects the extent of ultrasound-enhanced thrombolysis in an ex vivo porcine thrombosis model," Ultrasound Med Biol, vol. 39, no. 5, pp. 813-824, May 2013, doi: 10.1016/j.ultrasmedbio.2012.12.008.

43. S. Jeon, E.-Y. Park, W. Choi, R. Managuli, K. jong Lee, and C. Kim, "Real-time delay-multiply-and-sum beamforming with coherence factor for in vivo clinical photoacoustic imaging of humans," Photoacoustics, vol. 15, p. 100136, Sep. 2019, doi: 10.1016/j.pacs.2019.100136.

44. G. Matrone, A. S. Savoia, G. Caliano, and G. Magenes, "The Delay Multiply and Sum Beamforming Algorithm in Ultrasound B-Mode Medical Imaging," IEEE Transactions on Medical Imaging, vol. 34, no. 4, pp. 940-949, Apr. 2015, doi: 10.1109/TMI.2014.2371235.

45. M. Li, Y. Tang, and J. Yao, "Photoacoustic tomography of blood oxygenation: A mini review," Photoacoustics, vol. 10, pp. 65-73, Jun. 2018, doi: 10.1016/j.pacs.2018.05.001.

46. M.-L. Li et al., "Simultaneous Molecular and Hypoxia Imaging of Brain Tumors In Vivo Using Spectroscopic Photoacoustic Tomography," Proceedings of the IEEE, vol. 96, no. 3, pp. 481-489, Mar. 2008, doi: 10.1109/JPROC.2007.913515. 
47. L. J, E. C, D. D, and B. P, “In vitro measurements of absolute blood oxygen saturation using pulsed near-infrared photoacoustic spectroscopy: accuracy and resolution," Physics in medicine and biology, vol. 50, no. 18, Sep. 2005, doi: 10.1088/0031-9155/50/18/011.

48. J. D. Ferry and P. R. Morrison, "Preparation and Properties of Serum and Plasma Proteins. VIII. The Conversion of Human Fibrinogen to Fibrin under Various Conditions1,2," J. Am. Chem. Soc., vol. 69, no. 2, pp. 388-400, Feb. 1947, doi: 10.1021/ja01194a066.

49. L. Faxälv, P. Tengvall, and T. L. Lindahl, "Imaging of blood plasma coagulation and its propagation at surfaces," Journal of Biomedical Materials Research Part A, vol. 85A, no. 4, pp. 1129-1134, 2008, doi: 10.1002/jbm.a.31529.

50. S. Jia, Y. Zhang, T. Ma, H. Chen, and Y. Lin, "Enhanced Hydrophilicity and Protein Adsorption of Titanium Surface by Sodium Bicarbonate Solution," Journal of Nanomaterials, vol. 2015, p. e536801, Nov. 2015, doi: 10.1155/2015/536801.

51. J. Y. Chueh, A. K. Wakhloo, G. H. Hendricks, C. F. Silva, J. P. Weaver, and M. J. Gounis, "Mechanical Characterization of Thromboemboli in Acute Ischemic Stroke and Laboratory Embolus Analogs," American Journal of Neuroradiology, vol. 32, no. 7, pp. 1237-1244, Aug. 2011, doi: 10.3174/ajnr.A2485.

52. Y. Tang and J. Yao, "3D Monte Carlo simulation of light distribution in mouse brain in quantitative photoacoustic computed tomography," Quant Imaging Med Surg, vol. 11, no. 3, pp. 1046-1059, Mar. 2021, doi: $10.21037 /$ qims-20-815.

53. D. M. Dohan Ehrenfest, M. Del Corso, A. Diss, J. Mouhyi, and J.-B. Charrier, "Three-dimensional architecture and cell composition of a Choukroun's platelet-rich fibrin clot and membrane," $J$ Periodontol, vol. 81, no. 4, pp. 546-555, Apr. 2010, doi: 10.1902/jop.2009.090531.

54. J. Kim et al., "Laser-generated-focused ultrasound transducers for microbubble-mediated, dualexcitation sonothrombolysis," in 2016 IEEE International Ultrasonics Symposium (IUS), Sep. 2016, pp. 1-4. doi: 10.1109/ULTSYM.2016.7728473.

\section{Figures}




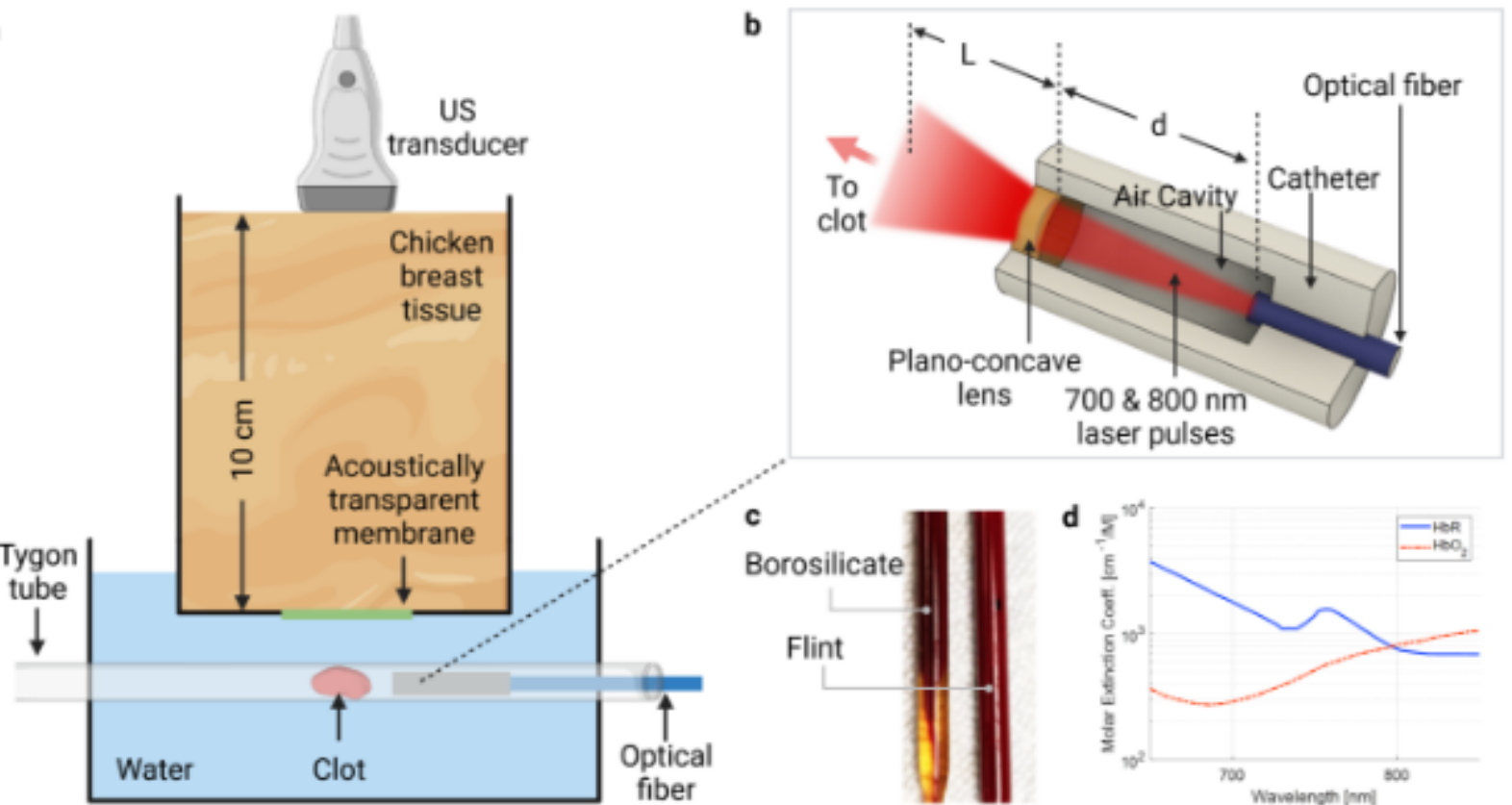

Figure 1. PACT of blood clot with intravascular light illumination. (a) Deep tissue clot characterization with photoacoustic computed tomography. A Tygon tube containing clot and catheter was immersed in water. A layer of ten-centimeter-thick chicken tissue was placed between the Tygon tube and the transducer. (b) Interior design of the catheter. The distance between catheter and clot L was $5 \mathrm{~mm}$. The length of cavity $\mathrm{d}$ was designed based on simulation results. (c) Picture of clot formed in borosilicate and flint Pasteur pipets. Only $\mathrm{CaCl}_{2}$ was added for coagulation. Serum appears in borosilicate pipets, indicating the clot retraction. (d) Absorption spectra of oxygenated $\left(\mathrm{HbO}_{2}\right)$ and deoxygenated $(\mathrm{HbR})$ hemoglobin.

\section{Figure 1}

PACT of blood clot with intravascular light illumination. (a) Deep tissue clot characterization with photoacoustic computed tomography. A Tygon tube containing clot and catheter was immersed in water. A layer of ten-centimeter-thick chicken tissue was placed between the Tygon tube and the transducer. (b) Interior design of the catheter. The distance between catheter and clot $L$ was $5 \mathrm{~mm}$. The length of cavity $d$ was designed based on simulation results. (c) Picture of clot formed in borosilicate and flint Pasteur pipets. Only $\mathrm{CaCl}_{2}$ was added for coagulation. Serum appears in borosilicate pipets, indicating the clot retraction. (d) Absorption spectra of oxygenated $\left(\mathrm{HbO}_{2}\right)$ and deoxygenated $(\mathrm{HbR})$ hemoglobin.

\section{Figure 2}

PA imaging of blood clot in tissue phantom. (a) Simulated beam fraction power with different air cavity length d. (b) Simulated beam profile with different air cavity length d. Without lens and air cavity, a narrower beam profile is observed. (c) PA image reconstructed with delay-and-sum (DAS) algorithm. Dotted lines indicate the Tygon tube boundaries. (d) PA image reconstructed with delay-multiply-and-sum (DMAS) algorithm. (e) US image indicates the thickness of chicken breast tissue and depth of the Tygon tube. (f) Overlayed PA and US images. 

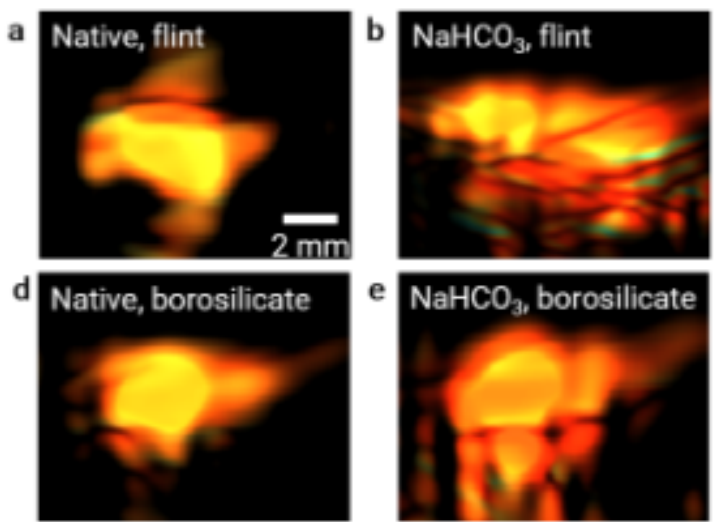

$\mathrm{NaHCO}_{3}$, borosilicate
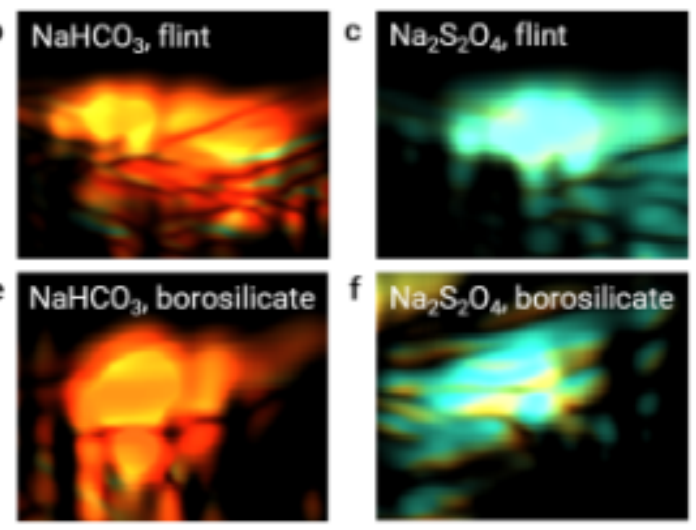

Oxygenation level

$100 \%$

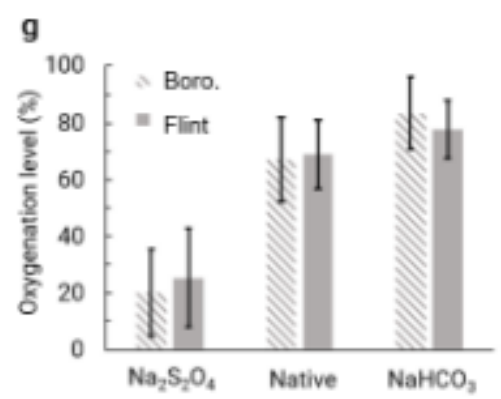

Figure 3. PACT of oxygenation saturation of hemoglobin $\left(\mathrm{sO}_{2}\right)$ of clot samples. (a-c) $s \mathrm{O}_{2}$ of clots stored in flint $\mathrm{Pasteur}$ pipets with (a) $\mathrm{CaCl}_{2}$ only (or native), (b) $\mathrm{CaCl}_{2}$ and $\mathrm{NaHCO}_{3}$, and (c) $\mathrm{CaCl}_{2}$ and $\mathrm{Na}_{2} \mathrm{~S}_{2} \mathrm{O}_{4}$ added to blood. (d-f) $5 \mathrm{O}_{2}$ of clots stored in borosilicate Pasteur pipets with (a) $\mathrm{CaCl}_{2}$ only, (b) $\mathrm{CaCl}_{2}$ and $\mathrm{NaHCO}_{3}$, (c) $\mathrm{CaCl}_{2}$ and $\mathrm{Na}_{2} \mathrm{~S}_{2} \mathrm{O}_{4}$ added to blood. (g) Averaged sO of clot samples. Error bars indicate the $\mathrm{s}_{2}$ variation within the clots.

\section{Figure 3}

PACT of oxygenation saturation of hemoglobin $\left(\mathrm{sO}_{2}\right)$ of clot samples. $(\mathrm{a}-\mathrm{c}) \mathrm{sO}_{2}$ of clots stored in flint Pasteur pipets with (a) $\mathrm{CaCl}_{2}$ only (or native), (b) $\mathrm{CaCl}_{2}$ and $\mathrm{NaHCO}_{3}$, and (c) $\mathrm{CaCl}_{2}$ and $\mathrm{Na}_{2} \mathrm{~S}_{2} \mathrm{O}_{4}$ added to blood. (d-f) $\mathrm{sO}_{2}$ of clots stored in borosilicate Pasteur pipets with (a) $\mathrm{CaCl}_{2}$ only, (b) $\mathrm{CaCl}_{2}$ and $\mathrm{NaHCO}_{3}$, (c) $\mathrm{CaCl}_{2}$ and $\mathrm{Na}_{2} \mathrm{~S}_{2} \mathrm{O}_{4}$ added to blood. (g) Averaged $\mathrm{sO}_{2}$ of clot samples. Error bars indicate the $\mathrm{sO}_{2}$ variation within the clots. 

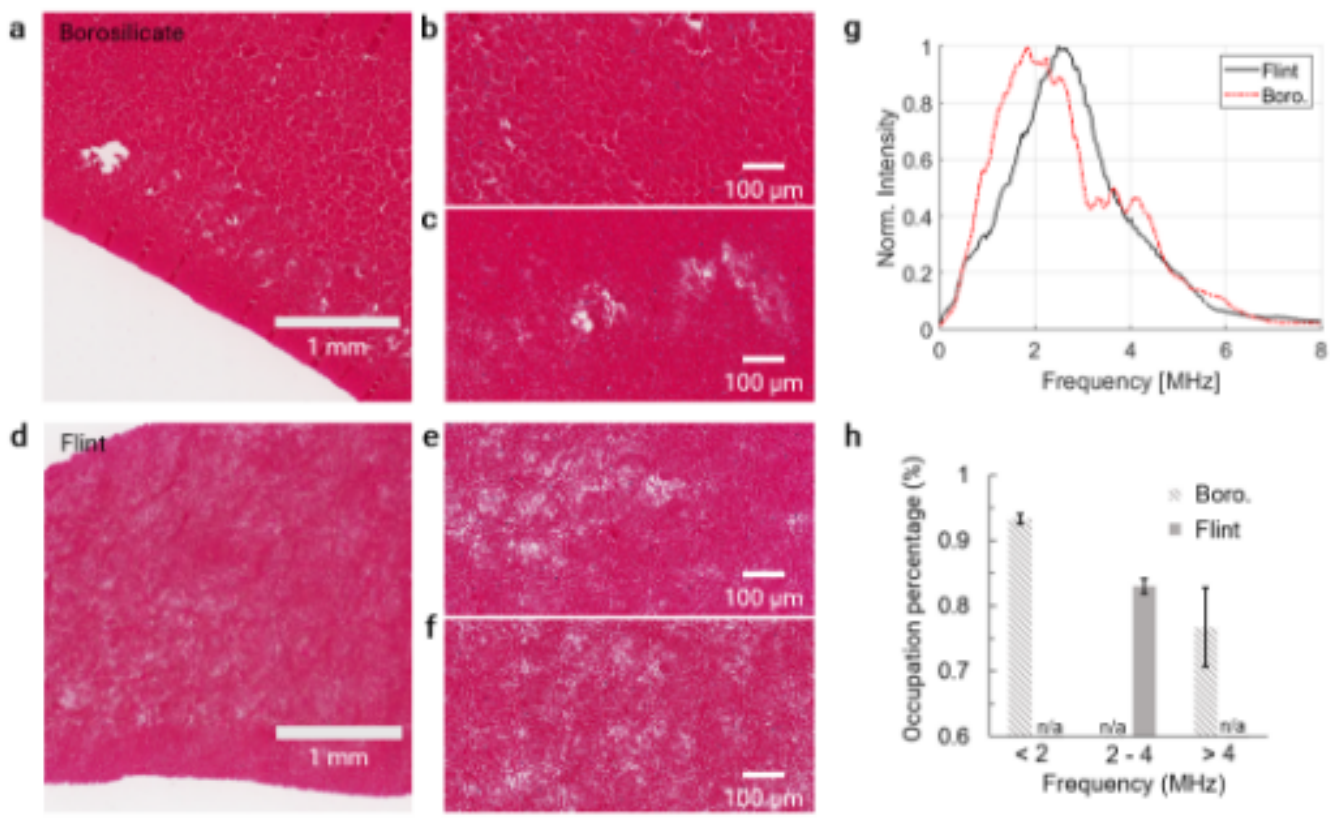

h

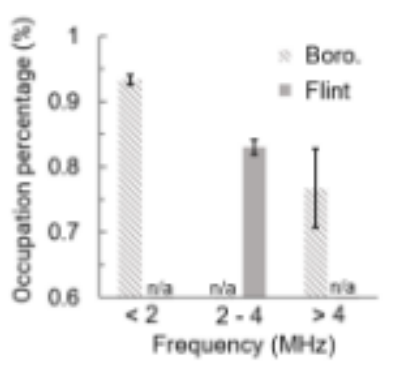

Figure 4. Histology and frequency spectrum analysis of blood clots with $\mathrm{CaCl}_{2}$ only. (a-c) $\mathrm{Histology}$ results of blood with $\mathrm{CaCl}_{2}$ in borosilicate Pasteur pipets. Retracted clot was expected. (d-f) Histology results of blood with $\mathrm{CaCl}_{2}$ in flint Pasteur pipets. Unretracted clot was expected. (g) Frequency spectrum of PA channel data acquired at $800 \mathrm{~nm}$. (h) Red blood cell occupation percentage as an indicator for clot retraction. Structural features that potentially associate with the frequency spectrum were identified, relevant regions were extracted from a, d, and the percentage of red blood cell pixels was calculated. The occupation percentage by red blood cells was associated with low $(<2 \mathrm{MHz})$, medium $(2-4 \mathrm{MHz})$, and high (>4 MHz) frequency

\section{Figure 4}

Histology and frequency spectrum analysis of blood clots with $\mathrm{CaCl}_{2}$ only. (a-c) Histology results of blood with $\mathrm{CaCl}_{2}$ in borosilicate Pasteur pipets. Retracted clot was expected. (d-f) Histology results of blood with $\mathrm{CaCl}_{2}$ in flint Pasteur pipets. Unretracted clot was expected. (g) Frequency spectrum of PA channel data acquired at $800 \mathrm{~nm}$. (h) Red blood cell occupation percentage as an indicator for clot retraction. Structural features that potentially associate with the frequency spectrum were identified, relevant regions were extracted from $a, d$, and the percentage of red blood cell pixels was calculated. The occupation percentage by red blood cells was associated with low ( $<2 \mathrm{MHz})$, medium $(2-4 \mathrm{MHz})$, and high $(>4 \mathrm{MHz})$ frequency bands. 
a
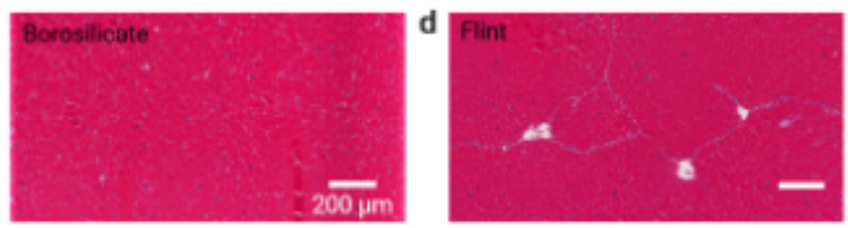

b
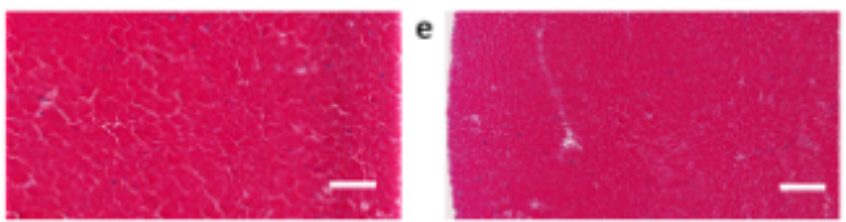

c
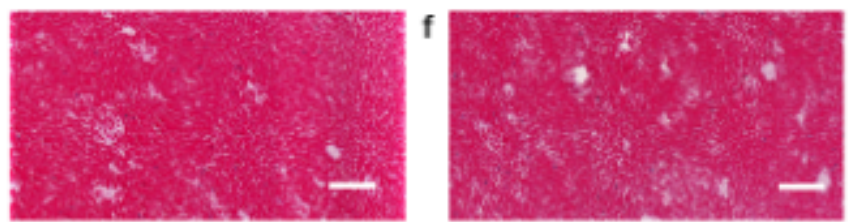

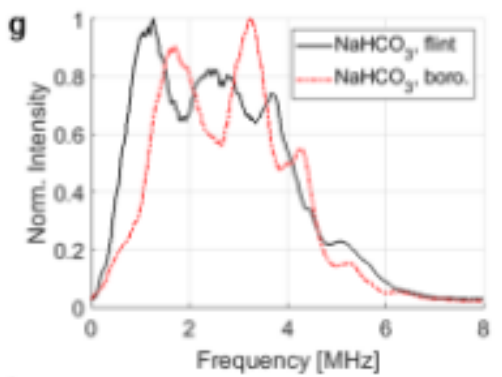

h

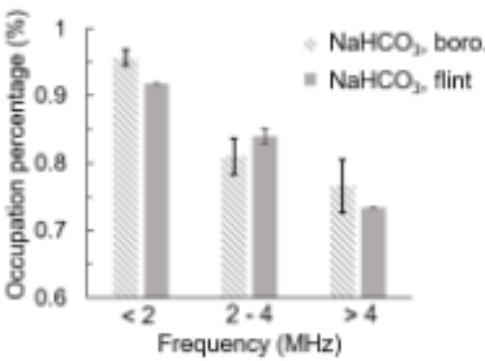

Figure 5. Histology and frequency spectrum analysis of blood clots with $\mathrm{CaCl}_{2}$ and $\mathrm{NaHCO}_{3}$. (a-c) Histology results of clots in borosilicate Pasteur pipets. (d-f) Histology results of clots in flint Pasteur pipets. (g) Frequency spectrum of PA channel data acquired at $800 \mathrm{~nm}$. (h) Red blood cell occupation percentage as an indicator for clot retraction.

\section{Figure 5}

Histology and frequency spectrum analysis of blood clots with $\mathrm{CaCl}_{2}$ and $\mathrm{NaHCO}_{3}$. (a-c) Histology results of clots in borosilicate Pasteur pipets. (d-f) Histology results of clots in flint Pasteur pipets. (g) Frequency spectrum of PA channel data acquired at $800 \mathrm{~nm}$. (h) Red blood cell occupation percentage as an indicator for clot retraction.

\section{Figure 6}

Histology and frequency spectrum analysis of blood clots with $\mathrm{CaCl}_{2}$ and $\mathrm{Na}_{2} \mathrm{~S}_{2} \mathrm{O}_{4}$. (a-c) Histology results of clots in borosilicate Pasteur pipets. (d-e) Histology results of clots in flint Pasteur pipets. (f) Frequency spectrum of PA channel data acquired at $800 \mathrm{~nm}$. (g) Red blood cell occupation percentage as an indicator for clot retraction. 
a

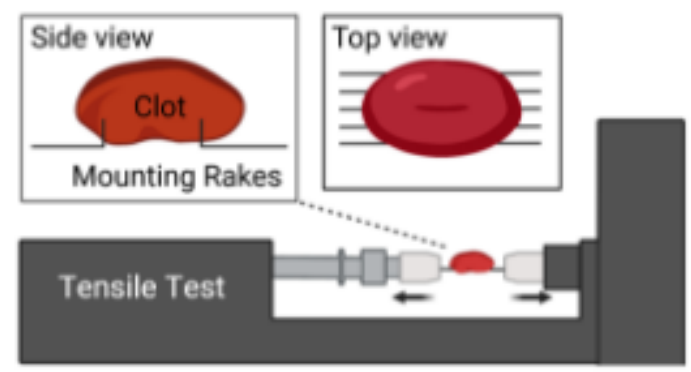

b 20

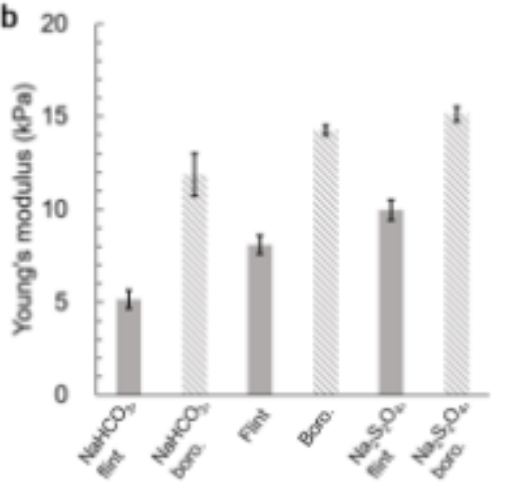

Figure 7. Tensile test of the blood clots. (a) Tensile test setup. Two rakes with five tungsten tines each were used at the ends of the cylindrical clot in the longitudinal directions. (b) Young's modulus of the clot samples estimated from the tensile test.

\section{Figure 7}

Tensile test of the blood clots. (a) Tensile test setup. Two rakes with five tungsten tines each were used at the ends of the cylindrical clot in the longitudinal directions. (b) Young's modulus of the clot samples estimated from the tensile test. 